COMPUTATIONAL METHODS IN SCIENCE AND TECHNOLOGY 4,57-69 (1998)

\title{
CONFIGURATION INTERACTION METHODS
}

\author{
JACEK KARWOWSKI ${ }^{1}$ AND GRZEGORZ PESTKA ${ }^{2}$ \\ Institute of Physics, M. Kopernik University \\ Grudziadzka 5, 87-100 Toruń, Poland \\ e-mail: 1 jka@phys.uni.torun.pl, ${ }^{2} g p @$ phys.uni.torun.pl
}

\begin{abstract}
In this article configuration interaction methods of solving the Schrödinger eigenvalue problem are reviewed. In particular computational aspects of the "traditional" and of the direct approach are briefly discussed. A special attention is given to the group-theory based formulations known as the unitary group approach and the symmetric group approach. Recent developments and implementations to relativistic theory of many-electron systems are also described.
\end{abstract}

\section{INTRODUCTION}

An TV-electron one-determinantal wavefunction corresponding to a closed shell molecule and based on the molecular orbital (MO) description of a single electron, defines an electronic configuration. The electronic configuration may be associated with the Hartree product corresponding to the one-determinantal wavefunction. To a given Hartree product of a closed shell system corresponds one Slater determinant and one electronic state of the molecule. The energy of this configuration is defined as the expectation value of the pertinent Hamiltonian. In this sense, the energy depends upon the choice of the orbitals. Usually it is assumed that orbitals derived from the Hartree-Fock equations are the most appropriate: they give the best variational one-determinantal approximation to the corresponding eigenfunction of the $\mathrm{N}$-electron Hamiltonian.

It has been realized already as early as in 1928 [1] that a considerable improvement of the results may be achieved by constructing the wavefunction as a linear combination of Slater determinants corresponding to several electronic configurations with coefficients determined by the Rayleigh-Ritz variational principle [2-4], The approach was named configuration interaction (CI) method. More recently this name has been extended to a broad family of methods in which many-electron wavefiinctions are represented as linear combinations of antisymmetrized products of orbitals, known as the configuration statefunctions (CSFs) [5-10], CSFs are usually adapted to various symmetries of the system. For example, the invariance of the Hamiltonian with respect to rotations in the spin space results in CSFs being eigenfunctions of the total spin operators $\hat{S}^{2}$ and $\hat{S}_{z}$. The same kind of invariance, but in the orbital space, causes that CSFs may be selected as eigenfunctions of the total orbital angular momentum operators, etc.

Various modifications of this approach resulted in most sophisti- cated computational algorithms. Some of these algorithms have been designed to include hundreds of millions of terms in the expansions [11, 12], The results based on the full CI (FCI) expansions, when all CSFs which may be constructed using a given set of orbitals are included, con- stitute benchmarks for many other methods. In fact, most of methods developed in quantum chemistry may be considered as approximations of the FCI results and a statement by Knowles and Handy, that "Full 
configuration interaction represents the ultimate goal of quantum chemistry" [12] does not seem to be entirely incorrect.

In this paper we give a brief review of the CI methods. Since several reviews on this subject have been recently published [13-15], we concentrate on these aspects of CI which were not adequately discussed in the previous works. In particular we stress some difficulties and limitations of the method. We also report on specific features of not very common implementations, as in the case of spin-dependent Hamiltonians.

\section{THE BASIC FORMULATION}

The basic formulation of all CI methods is derived from a theorem usually associated with Lôwdin [5]. The theorem says that if a set of orbitals $\phi_{j}, j=1,2, \ldots$ is complete in the one-electron space of square-integrable functions then the set of all $\mathrm{N}$-electron Slater determinants $\Psi(1,2, \ldots, N)_{L}$ which may be constructed using these orbitals is complete in the space of squareintegrable antisymmetric $N$-electron functions. As a consequence, each antisymmetric wavefunction $\Psi(1,2, \ldots, N)$ describing a bound state (i. e. being square-integrable) may be expanded as

$$
\Psi=\sum_{L} C_{L \psi L} .
$$

In more general terms one can say that $\Psi$ is an element of an antisymmetric space spanned by the $\mathrm{N}$-electron determinants. In computational implementations this space has to be finite-dimensional. Consequently, the set of orbitals $\phi_{j}$ has to be finite, i. e. $j=1,2, \ldots, K$. The corresponding $N$-clectron space is called the full CI space. Its dimension is equal to $\left(\begin{array}{l}2 K \\ N\end{array}\right)$ - the number of $N$-electron Slater determinants. The FCI space may be considered to be the model space in which the eigenvalue problem of the pertinent Hamiltonian has to be solved. In the model space the general eigenvalue problem

$$
\hat{H} \Psi_{M}=E_{M} \Psi_{M}
$$

is reduced to

$$
\sum_{J}\left[\left\langle\Psi_{I}|\tilde{H}| \Psi_{J}\right\rangle-\delta_{I J} \tilde{E}_{M}\right]\left\langle\Psi_{J} \mid \Psi_{M}\right\rangle=0
$$

where

$$
\tilde{H}=\sum_{A B}\left|\Psi_{A}\right\rangle\left\langle\Psi_{A}|\tilde{H}| \psi_{B}\right\rangle\left\langle\psi_{B}\right|
$$

is the projection of $\hat{H}$ onto the model space.

One has to remember that the spectrum of the matrix eigenvalue problem given by Eq. (3) is much poorer than the one of Eq. (2). In particular, all states belonging to the continuum as well as the resonances cannot be correctly described by Eq. (3). In practical terms, only the ground state and several lowest excited states may be reproduced with a reasonable accuracy by solving Eq. (3). Also many operators cannot be correctly represented in the full CI space. Perhaps the most striking example is a representation of the commutation relation

$$
i[\hat{\mathbf{p}}, \hat{\mathbf{x}}]=1 \text {. }
$$


Using the resolution of the identity in the model space:

$$
\sum_{A}\left|\psi_{A}\right\rangle\left\langle\psi_{A}\right|=1
$$

one can easily show that the trace of the left-hand side of Eq. (5) is equal to 0 while the trace of the right-hand side is equal to the dimension of the space. This contradiction results from the fact, that the momentum operator can never be represented correctly in a discrete space.

\section{THE MODEL SPACE}

If the Hamiltonian is spin-independent, its eigenvalue problem in the FCI space may be factorized with respect to the total spin operators. In the most common approach the spin and the orbital degrees of freedom of electrons are coupled to form the one-electron spin-orbital space. The basis in this space is formed by a set of $2 K$ spinorbitals. In the next step the $N$-electron basis of CSFs is constructed as spin-adapted combinations of antisymmetrized products of spinorbitals. The set of CSFs corresponding to a given pair of the total spin quantum numbers (related to the square of the total spin operator and to its projection) form a basis in the spin-adapted CI space. Many methods of construction of CSFs are described in a very good monograph by Pauncz [16].

The observation that the FCI space is invariant with respect to unitary trans formations of the one-electron basis and that the Hamiltonian projected onto the FCI space may be expressed in terms of the unitary group generators lead J. Paldus to the formulation of the unitary group approach (UGA) to the CI method [17]. The Gelfand tableau representation of the $N$-electron basis provided not only a convenient unique scheme for the labelling of CSFs but also allowed for construction of a graphical representation of the spin-adapted CI space by Shavitt [18] and formulation of what is known as the graphical unitary group approach (GUGA).

Another approach, which may be traced back to early works of Kotani et al. [19], has been developed in works of Hams [20], Ruedenberg [21], Gerrat [22] and of the present author [23, 24], In this approach, referred to as the symmetric group approach (SGA) [10], the $\mathrm{N}$-electron spin and orbital spaces are constructed independently. They are coupled at the

$\mathrm{N}$-electron level to form the spin-adapted CI space. In effect the computational algorithms based on the SGA are simpler and more efficient than these derived from UGA [25]. Also the graphical representations of the model spaces are more compact and transparent. The resulting graphical approach is referred to as the symmetric group graphical approach (SGGA) [10].

\subsection{Symmetric group approach}

In SGA the $N$-electron basis in the spin space is formed by the eigenvectors of the total spin operators:

$$
\hat{S}^{2}|S M, l\rangle=S(S+1)|S M, l\rangle, \hat{S}_{z}|S M, l\rangle=M|S M, l\rangle, l=1,2, \ldots, f
$$

where

$$
f \equiv f(S, N)=\frac{2 S+1}{N+1}\left(\begin{array}{c}
N+1 \\
N / 2-S
\end{array}\right)
$$


The symmetric group $S_{N}$ composed of $N$ ! permutations is an invariance group of $\hat{S}^{2}$ and $S_{z}$. Therefore eigenfunctions of $\hat{S}^{2}$ and $\hat{S}_{z}$ form bases for irreducible representations of $S_{N}$, i. e.

$$
\hat{P}|S M, l\rangle=\sum_{j=1}^{f} V_{S}^{N}(P)_{j l}|S M, j\rangle,
$$

where $V_{S}^{N}(P)_{j l}=\langle S M, j|\hat{P}| S M, l\rangle$ is a matrix element of the irreducible representation of $S_{N}$ corresponding to a two-row Young shape with $x=1 / 2 N+S$ boxes in the first row and $y=1 / 2 N-S$ boxes in the second row.

The $\mathrm{N}$-electron basis in the orbital space is formed by a set of Hartree products:

$$
\Psi_{\lambda}^{0}(\mathbf{r})=\prod_{i=1}^{N} \phi_{\lambda i}\left(\mathbf{r}_{i}\right)
$$

Each Hartree product corresponds to an orbital configuration $\lambda=\left\{\lambda_{1}, \lambda_{2}, \ldots, \lambda_{N}\right\}$. The Pauli principle causes that in a given configuration an orbital may be doubly occupied (then it is referred to as a double and appears twice in the product), singly occupied (it is referred to as a single and appears once in the product) or empty (does not appear in the product). If $d_{\lambda} / s_{\lambda}$ denote the number of doubles/singles in $\lambda$, then

$$
s_{\lambda}+2 d_{\lambda}=N
$$

The basis in the orbital space is composed of all products (10) which fulfill condition (11).

The Cartesian product of the $N$-electron spin- and orbital-spaces gives the spin-adapted FCI space. Its dimension is given by the Weyl-Paldus formula [26]

$$
D(N, K, S)=\frac{2 S+1}{K+1}\left(\begin{array}{c}
K+1 \\
N / 2-S
\end{array}\right)\left(\begin{array}{c}
K+1 \\
N / 2+S+1
\end{array}\right)
$$

Though considerably smaller than the dimension of the FCI space, it grows up very rapidly with an increase of $N$ and $K$. This behaviour of $D$ (for $S=0$ ) is shown in Fig. 1. As one can see, even in very small orbital bases, say $K=2 N$, the dimensions of the spin-adapted FCI space become prohibitively large already for systems with about 10 electrons. Therefore, in all formulations of $\mathrm{CI}$ methods a reasonable truncation of the $\mathrm{CI}$ expansion plays most important role. This leads to many different restricted $\mathrm{CI}$ methods.

\section{2. The McDonald theorem}

Relations between results of a full and a restricted CI calculations are given by the McDonald theorem [5,27]: Let the CI space be spanned by J basisfunctions and let the CI energies be

$$
E_{1}^{(J)} \leq E_{2}^{(J)} \leq \ldots \leq E_{J}^{(J)}
$$


Fig. 1. Dimension of the spin-adapted FCI space corresponding to $S=0$ as a function of $K$ and $N$. $A$ line labelled by $k$ corresponds to the dimensions of the FCI space equal to $10^{k}$

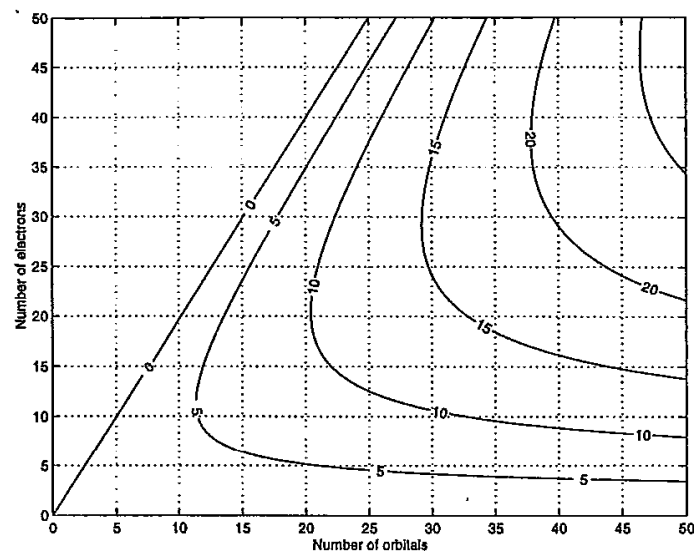

as the dimension of the CI space is increased by adding an additional basisfunction, eigenvalues $E_{p}^{(J+1)}$ of the $(J+1)$ - dimensional problem satisfy the inequalities:

$$
E_{p-1}^{(J)} \leq E_{p}^{(J+1)} \leq E_{p}^{(J)}
$$

and as the set of one-electron functions approaches completeness, the FCI solutions approach the exact solutions of the pertinent eigenvalue equation. From this theorem results that each eigenvalue of the CI matrix is an upper bound to the corresponding discrete eigenvalue of the Hamiltonian.

\section{3. Graphical representations}

Model spaces in which the basis vectors are labelled by sequences of one-electron occupation numbers (i. e. by either orbital or spinorbital configurations) may be conveniently represented in a graphical way. The model space is either described by one or by two graphs composed of a network of paths. Each of the paths describes one $N$-electron basis function. The path is composed of either $K$ or $2 K$ arcs, depending upon the mode of the description. The slope of the arc describes the one-electron occupation number. Then, in the spinorbital description we have the two-slope graphs (since the occupation numbers may be equal to either 0 or 1 ). In the orbital description the occupation numbers may be equal to 0,1 and 2 . Therefore in this case we have three-slope orbital graphs. If the FCI; space is split into spin-adapted subspaces then the basis in each subspace is composed of eigenfunctions of the total spin operators $\hat{S}^{2}$ and $\hat{S}_{z}$. Such spaces may be represented by four- slope graphs [18], in which both orbital and spin structure of the basis functions are described by each path. This representation of the model spaces is specific for the unitary group approach to theory of many- electron systems. In SGA, where the model space is constructed as an antisymmetrized direct product of the orbital space and of the spin space, each of these spaces is represented by an independent graph [10], The orbital space is then described by a three-slope orbital graph and the spin space - by a two-slope spin graph also known as the van Vleck's branching diagram (Pauncz 1979) [16], A detailed analysis of pro- 
perties of the model spaces and a description of different ways of their graphical representations may be found in a monograph by Duch [28],

\section{4. The orbitals}

Usually orbitals for CI calculations (referred to as the "molecular" orbitals) are taken as linear combinations of some localized orbitals $\left\{\varphi_{c}\right\}_{c=1}^{K^{\prime}}$, with $K^{\prime} \geq K$, referred to as "atomic" though frequently they are centered in properly selected points rather than in the atomic nuclei. Coefficients of these combinations are, most commonly, determined in a Roothaan-Hartree-Fock SCF procedure.

Another choice is to use the natural orbitals [5], The natural orbitals are eigenfunctions of the first-order density matrix corresponding to a given wavefunction and assure the fastest convergence of the CI expansion corresponding to this wavefunction. The difficulty in using these orbitals is that the density matrix and, therefore, also the $\mathrm{CI}$ wavefunction is required to calculate the natural orbitals. Thus, they can be obtained only after the CI calculation is completed. Several methods allowing to construct approximate natural orbitals have been developed and successfully applied [29-31]

Procedures in which both coefficients in the CI expansion and in the molecular orbitals are simultaneously optimized using the variational principle are known as multiconfiguration self consistent field (MC-SCF) methods. They lead to rather compact expansions of the wavefunctions, however frequently cause convergence problems. A pedagogical review of the MC-SCF techniques has been published by McWeeny and Sutcliffe [32].

The FCI space is invariant with respect to linear transformations of the orbitals. Therefore, if $K^{\prime}=K$ then the FCI results are the same for any set of the molecular orbitals constructed from a given set of atomic orbitals. However this is not the case for a limited (restricted) CI. This behaviour of the CI results is illustrated in Fig. 2.

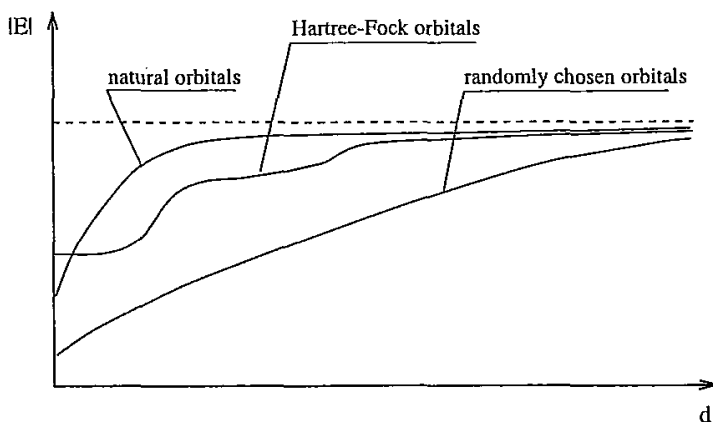

Fig. 2. Pattern of convergence of the CI expansions for various kinds of molecular orbitals constructed from the same set of primitive atomic orbitals. The absolute value of the energy $|E|$ is plotted versus the length of the CI expansion $d$, ordered according to the excitation multiplicity

If the MO basis consists of the Roothaan-Hartree-Fock orbitals, then the CI expansion limited to a single CSF is the Roothaan-Hartree-Fock $N$-electron wavefunction and it gives the RoothaanHartree-Fock energy $\left(E_{H F}\right)$. With this choice of MOs the CI expansion is usually dominated by one or by a few CSFs - the reference CSFs. The orbitals used to construct the reference CSFs are referred to as the occupied or internal orbitals - as distinct from the virtual or external 
orbitals, which do not appear in the reference CSFs. The internal orbitals which are doubly occupied in all reference configurations are "frozen" in the space of the reference CSFs. The remaining internal orbitals, are distinguished as the active orbitals. Then, the orbital space is divided into two major subspaces - the internal space and the external space. The internal space consists of the doubly occupied space and the active space.

\section{CI EXPANSIONS}

The most commonly used configuration selection scheme is based on the excitation multiplicity criterion. One starts with the SCF wavefunction as the reference configuration and adds all configurations in which no more than a predetermined number, say $m$, of the occupied orbitals have been replaced by the virtual ones ( $m=N$ for the FCI). The simplest version of this scheme, when only single and double replacements (excitations) are allowed is referred to as singly and doubly excited CI (SD CI). The SD CI is very frequently used because of its formal simplicity. The fact that the singly and doubly excited configurations are the only ones which interact directly (have non-zero matrix elements) with the reference configuration may justify this kind of truncation of the CI expansion if the SCF wavefunction gives a good zeroth-order description, as it frequently happens for small molecules, close to their equilibrium geometries. However, with growing dimension of the molecule, SD CI becomes increasingly poor due to its inherent limitations known as the size-consistency error. Most discouraging is the following theorem valid for a system of $n$ spatially separated electron pairs (e. g. for $n$ hydrogen molecules): Any truncation of the CI expansion to a finite order of excitation leads, as $n$ tends to infinity, to zero overlap with the exact wavefunction and a zero valuefor the calculated correlation energy per localized electron pair [33].

Another, much more efficient way to improve the CI expansion, is using several reference configurations with SD CI out of all of them. The resulting multi-reference (MR) SD CI expansion may reach any arbitrary accuracy by enlarging the number of the reference configurations. Several extrapolation schemes aimed at recovering the FCI energies from a series of MR SD CI calculations have been developed and claimed to be veiy efficient (e. g., nearly exact recovering the FCI energy for $\mathrm{H}_{2} \mathrm{O}$ in double-zeta basis using only $5 \%$ of the corresponding spin-adapted FCI expansion [34]).

A reasonable compromise between accuracy and compactness of the CI expansion is based on the idea of the complete active space (CAS) $[35,36]$. CAS is the $N$-electron, spin- and symmetry-adapted space spanned by all CSFs which may be constructed using the active orbitals. In the CAS SCF method the wavefunction is expressed as a linear combination of the CAS basis functions. Both the mixing coefficients and the orbitals are subject to a multi-configuration SCF optimization procedure. The optimized CAS expansion may be supplemented by a set of configurations singly and doubly excited relatively to the ones in the active (internal) space. Contributions from the doubly excited configurations forming the external space, may be included in a very simple way. This observation is of a fundamental importance in constructing very efficient CI programs [8, 37]. 
An inadequate treatment of the singularity in the electron-electron interaction operator is, to a large extent, responsible for the slow convergence of CI expansions [38-40], Therefore explicitly including the interelectronic distance to the CI wavefunction may considerably reduce the necessary length of the expansion. The resulting method, known as the Hylleraas-CI [41, 42], leads to very high accuracy and compact wavefunctions. Yet, due to numerical difficulties, it has been applied to very small systems only. Very recently a most successful approach based on expansions in terms of the exponentially correlated Gaussians has been developed by Cencek et al. [43]. This method gave the most accurate results for molecules with $N<5$ (the total energy error is smaller by two orders of magnitude than in the most accurate CI calculations).

Another difficulty is associated with a description of the states with many open shells. If out of $N$ electrons $s$ belongs to an open shell then there are $2^{s}$ different Slater determinants corresponding to this electronic configuration. For a given pair $(S, M)$ of the total spin quantum numbers one may construct $f(S, s)$ eigenfunctions of the total spin operators. Then, for a given orbital configuration, there are $f(S, s)$ different CSFs and, consequantly $f(S, s)$ different electronic states. This number, small in the case of small $s$, grows up very rapidly when $s$ increases. For example, $f(0,2)=f(1,2)=1, f(0,4)=2, f(1,4)=3$, but $f(0,20)=16796, f(1,20)=41990$ and the total number of Slater determinants for 20 open shells is $2^{20}=1048$ 576. As one can see, an inclusion of many-open-shell configurations in the CI procedure leads to serious computational difficulties [44],

\section{THE HAMILTONIAN MATRIX ELEMENTS}

In general, the $N$-electron Hamiltonian contains one- and two-body interactions and explicitly depends upon spin. It can be expressed as

$$
\hat{H}=\hat{H}^{0}+\hat{H}^{1}
$$

where $\hat{H}^{0}$ is the spin-independent and $\hat{H}^{1}$ - the spin-dependent operator. More specifically,

$$
\hat{H}^{0}=\sum_{i=1}^{N} \hat{h}_{1}(i)+\sum_{i>j}^{N} \hat{h}_{2}(i, j),
$$

where $\hat{h}_{1}$ includes the kinetic energy and interactions of the electron with an external field (usually with the electrostatic field of the nuclei), and

$$
\hat{h_{2}}(i, j)=\frac{1}{r_{i j}}
$$

describes the electron-electron interactions. The spin-dependent part may be represented as

$$
\hat{H}^{1}=\sum_{i=1}^{N} \hat{h}_{1}^{\sigma}\left(\sigma_{i}\right) \hat{h}_{1}^{u}(i)+\sum_{i>1}^{N} \hat{h}_{2}^{\sigma}\left(\sigma_{i}, \sigma_{j}\right) \hat{h}_{2}^{u}(i, j),
$$

where the superscripts $\sigma$ and $u$ refer, respectively, to the spin- and the space-dependence. For simplicity, the sums in the last equation are extended over the electron coordinates only rather than over all components of the pertinent spin operators. 
In most general terms, the Hamiltonian matrix elements may be expressed as

$$
H_{m n}^{(\lambda \mu)}=\sum_{i j}^{K} \Gamma_{\lambda m, \mu n}^{i j}(i \mid j)+\frac{1}{2} \sum_{i j k l}^{K} \Gamma_{\lambda m, \mu n}^{i j k l}(i j \mid k l),
$$

where $(i \mid j)$ and $(i j \mid k l)$ denote, respectively, one- and two-electron integrals in the orbital basis and $\Gamma_{\lambda m, \mu n}^{i j}$ and $\Gamma_{\lambda m, \mu n}^{i j k l}$ are refered to as the coupling constants. The coupling constants have also an alternative meaning: they are, respectively, the first- and the second-order reduced density matrix elements, if $(\lambda m)=(\mu n)$, or reduced transition matrix elements, otherwise. The coupling constants may be expressed as

$$
\Gamma=\Gamma^{0}+\Gamma^{1}
$$

where $\Gamma^{0}$ stands for the contribution due to $\hat{H}^{0}$ and $\Gamma^{\mathrm{l}}$ - due to $\hat{H}^{1}$. As one can show [10, 45] the coupling constants may be expressed as

$$
\begin{gathered}
\Gamma_{\lambda m, \mu n}^{0^{j}}=(-1)^{p} \alpha_{\lambda \mu} \eta(i, j) V_{S}^{N}(P)_{m n} \delta\left(S, S^{\prime}\right) \delta\left(M, M^{\prime}\right) \\
\Gamma_{\lambda m, \mu n}^{0^{j k l}}=(-1)^{p} \alpha_{\lambda \mu} \eta(i, j, k, l) V_{S}^{N}(P)_{m n} \delta\left(S, S^{\prime}\right) \delta\left(M, M^{\prime}\right) .
\end{gathered}
$$

and

$$
\begin{gathered}
\Gamma_{\lambda m, \mu n}^{1^{j j}}=(-1)^{p} \alpha_{\lambda \mu} \eta(i, j)\left\langle S M, m\left|\hat{P} h_{1}^{\sigma}\left(\sigma_{j \mu}\right)\right| S^{\prime} M^{\prime}, n\right\rangle \\
\Gamma_{\lambda m, \mu n}^{1 / k k l}=(-1)^{p} \alpha_{\lambda \mu} \eta(i, j, k, l)\left\langle S M, m\left|\hat{P} h_{2}^{\sigma}\left(\sigma_{j \mu}, \sigma_{l \mu}\right)\right| S^{\prime} M^{\prime}, n\right\rangle
\end{gathered}
$$

where $\alpha_{\lambda \mu}=2^{q 2}$ with $q=0,1,2,3,4, \eta(i, j)=0,1,2$ and $\eta(i, j, h, l)=0,1,2,4$, depending upon the occupation numbers of the orbitals involved. The coefficients $\eta$ vanish, unless either $\hat{P}=\hat{P}_{0}$ or $\hat{P}=\hat{P}_{0}(i, k)$, where $\hat{P}_{0}$ is the permutation which brings orbitals of $\lambda$ into maximum coincidence with orbitals of $\mu$ (referred to as the line-up permutation) and $(i, k)$ denotes a transposition $[10,45]$,

\section{STRATEGY OF CI CALCULATIONS}

After the explicit expressions for the Hamiltonian matrix elements are known (Eqs. (19)-(24)), solving Eqs. (3) may be considered as a simple algebraic exercise: construct the CI matrix and then find its eigenvalues and eigenvectors using any of the standard methods of diagonalization. This approach, known as the traditional CI method, may easily be applied when the number of orbitals is small enough (say 20) to keep all two-electron integrals in the computer memory as a random access file. Besides, the Hamiltonian matrix has to be stored. As one can easily see, the number of two-electron integrals is approximately equal to $\mathrm{K}^{4} / 8$ and already for $K=100$ the number of two-electron integrals exceeds $10^{7}$ and becomes prohibitively large. Besides, though the majority of the Hamiltonian matrix elements vanish, checking whether a matrix 
element is equal to 0 or not, involves a comparison of the two configurations involved. This comparison, already for CI matrices of dimension 1000, take more than $90 \%$ of the total time for its construction. A method which is free of these deficiencies has been formulated by Roos [46] and is known as the direct $C I$ (DCI) method.

The main part of all iterative methods of solving a matrix eigenvalue problem is multiplication of the Hamiltonian matrix by its approximate eigenvector, i. e. finding

$$
\mathbf{B}^{(p+1)}=\mathbf{H} \mathbf{C}^{(p)},
$$

where the superscripts refer to the iteration numbers. In DCI solving matrix eigenvalue problem and evaluation of matrix elements is combined into a single algorithm. The product vector $\mathrm{B}^{(\mathrm{p}+1)}$

is here constructed directly from a list of one- and two-electron integrals, without constructing H. This avoids the auxiliary storage problems and dramatically reduces the data transferring tasks. The core of a DCI algorithm may be summarized as follows. Given the orbital indices $i, j, k, l$; find all pairs of configurations $\lambda, \mu$ for which the coupling constants $\Gamma_{\lambda m, \mu n}^{i j k l}$ do not vanish; determine values of the coupling constants; multiply them by the appropriate integrals and by the $(\mu n)$ component of $\mathbf{C}$; increment the $(\lambda \mathrm{m})$ component of $\mathbf{B}$ by the resulting product. As one can see, a single coupling constant corresponding to a given $i, j, k, l$ set of indices, may contribute to many components of $\mathbf{B}$ and $\mathbf{C}$. Therefore a random access to these vectors is necessary, i. e. they should be kept in the core memory.

Formulation of graphical approaches to CI became crucial to the final success of the DCI methods. In these approaches, instead of individual pair's of configurations, the entire spaces are handled. In both GUGA and SGGA a matrix element may be associated with a loop formed by two paths in the pertinent graph representing the model space. However in GUGA one loop corresponds to one matrix element while in SGGA a single loop describes, in general, an array of matrix elements. In SGA the CI basis is described by two independent graphs: the orbital graph and the spin graph. All matrix elements corresponding to a pair of orbital configurations $|\lambda\rangle$ and $|\mu\rangle$ are represented by the same loop formed by the corresponding paths in the orbital graph. Only some easily recognizable loops lead to non-zero matrix elements. The orbital indices $i, j, k, l$ define shapes of the non-zero loops and, in consequence, allow to find all pans of configurations coupled by $\Gamma_{\lambda m, \mu n}^{i j k l}$ and the corresponding permutations $\hat{P}_{0}$. Hence, the non-zero contributions to the CI matrix may be identified by recognizing specific patterns in the orbital graph. The orbital graph gives a symbolic form of the coupling constants. Their numerical values may be derived from the spin branching diagram. In effect not only calculating zeros is avoided but also, if several pairs of configurations lead to the same matrix element, then the element is calculated only once. If the Yamanouchi-Kotani spin functions are used [16, 19], then $\mathbf{U}_{S}^{N}$ matrices may be constructed directly from the spin graph [10], A careful optimization of this algorithm allows the computation of the representation matrices with a speed similar to that of a reading them from a file. This structure of the algorithm removes one of the main bottlenecks of many CI programs - constructing and storing a file containing all infor- mation about the structure of the CI matrix, the so called formula tape. A compact and transparent representation of the orbital space allows 
for various kinds of optimization - from optimizing ways of dealing with the external part of the orbital space, as discussed in papers [8] and [10], to algorithms in which blocks of the coupling constants are processed at the same time [25] or the ones in which graphs are cut into two parts processed independently and then combined together again [47],

\section{SPIN-DEPENDENT CI}

A similar approach may be applied also to spin dependent Hamiltonians. However the degree of complication is here slightly higher. Additionally to the representation matrices of $S_{N}$, matrices that depend upon the form of the spin-dependent part of the Hamiltonian appear in the coupling constant expressions (cf. Eqs. 21-24). A complete set of spin-dependent Hamiltonian matrix element formulae met in CI calculations have recently been derived by Flocke et al. [45], A general UGA spin-dependent theory has been formulated by Gould and Chandler [48]. If the Hamiltonian depends explicitly upon spin, then the total spin operators, in general, are not constants of the motion and the Hamiltonian matrix is not block-diagonal with respect to the total spin quantum numbers. However it is convenient to construct the CI procedure in a basis of spinadapted CSFs. Since in SGA the orbital graph is independent of the spin it is irrelevant whether matrix elements are associated with the same or with different total spin eigenstates. In particular, in the integral-driven direct CI for each set of orbital indices defining a molecular integral, the set of loops in the orbital graph and the pertinent permutations are identified in exactly the same way as in the case of spin-independent operators. Next, in the spin-independent case the appropriate representation matrices of are taken in order to construct the coupling constants. In the spin-dependent case, the coupling constant matrices are constructed according to Eqs. (23) and (24), for all pairs of the total spin quantum numbers taken into account. The coupling constant matrices have a block struture visualised in Fig. 3. It is composed of the blocks located in the diagonal part of the matrix which correspond to the coupling constants labelled by a specific value of $S$ and the off-diagonal blocks which couple states with different $S$ values.

Fig. 3. Structure of the coupling constant matrix corresponding to a given orbital configuration, when the $N$-electron CSFs are chosen to be eigenfunctions of $\hat{S}^{2}$ and $\hat{S}_{z}$

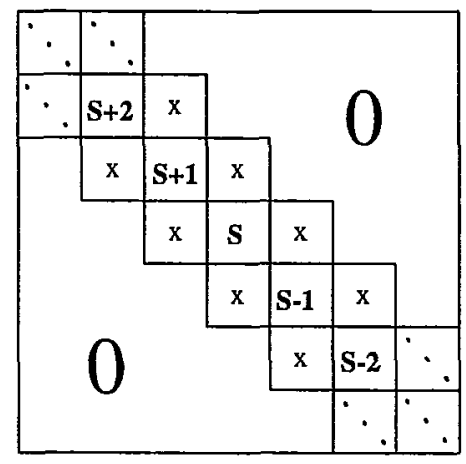

The coupling between different diagonal blocks is caused by spin-dependent terms only. In different stages of approximation one may include different degrees of coupling between $S$ and $S^{\prime}$. In the lowest approximation $S^{\prime}=S$, then $\left|S-S^{\prime}\right|=1$ are included, etc. The only essential 
difference between the direct CI algorithms for the cases of spin-dependent and spin-independent operators is in the form of the blocks of the $N$-electron integrals over spin variables. This allows for an easy construction of a relativistic, Pauli-type CI codes on a base of non-relativistic SGA-CI programs. Such a program has recently been developed as a part of the OPENMOL project [49],

\section{CONCLUDING REMARKS}

Majority of the standard methods of quantum chemistry may be cosidered as approximate ways of solving the FCI problem. They comprise a large variety of models ranging from different kinds or restricted CI calculations to methods based on the perturbational expansions, such as the many-body perturbation theory (MBPT) and the coupled clusters (CC) method. Broad reviews of all these approaches may be found in books edited by Wilson [50] and by Wilson and Diercksen [51].

\section{Acknowledgements}

This work has been supported by the Polish KBN under projects No. 2 P03B 12614 and 2 P03B 02312.

\section{References}

[1] E. A. Hylleraas, Z. Phys., 48, 469 (1928).

[2] S. F. Boys, Proc. Roy. Soc. (London), A217 136, 235 (1953).

[3] R. G. Parr, D. P. Craig, I. G. Roos, J. Chem. Phys., 18, 1561 (1950).

[4] A. Meckler, J. Chem. Phys., 21, 1750 (1953).

[5] P. -O. Löwdin, Phys. Rev., 97, 1509 (1955).

[6] I. Shavitt, in Methods ofElectronic Structure Theory, ed. H. F. Schaefer III (Plenum, New York, 1977), p. 189.

[7] B. O. Roos, P. E. M. Siegbahn, in Methods of Electronic Structure Theory, ed. H. F. Schaefer III (Plenum, New York, 1977), p. 277.

[8] V. R. Saunders, J. H. van Lenthe, Mol. Phys., 48, 923 (1983).

[9] M. Robb, U. Niazi, Comp. Phys. Reports, 1, 128 (1985).

[10] W. Duch, J. Karwowski, Comp. Phys. Reports, 2, 94 (1985).

[11] J. Olsen, B. O. Roos, P. Jorgensen, H. J, Aa. lensen, J. Chem. Phys., 89, 2185 (1988).

[12] P. J. Knowies, N. C. Handy, J. Chem. Phys., 91, 2396 (1989).

[13] W. Duch, J. Mol. Structure (Theochem), 234, 27 (1991).

[14] J. Karwowski, in Methods in Computational Physics, eds. G. H. F. Diercksen and S. Wilson (Plenum, New York, 1992), p. 65.

[15] J. Karwowski, in Computational Chemistry. Structure, Interactions and Reactivity, Part A, ed. S. Fraga (Elsevier, Amsterdam 1992), p. 197.

[16] R. Pauncz, Spin Eigenfunctions. Construction and Use, (Plenum, New York 1979).

[17] J. Paldus, in Theoretical Chemistry, Advances and Perspectives, vol. 2. eds. H. Eyring and D. G. Henderson (Academic Press, New York 1976) p. 131.

[18] I. Shavitt, Int. J. Quantum Chem., Sil, 131 (1977); S12, 5 (1978).

[19] M. Kotani et al., Table of Molecular Integrals (Maruzen, Tokyo 1955).

[20] F. E. Harris, J. Chem. Phys., 46, 2769 (1967); 47, 1047 (1967).

[21] K. Ruedenberg, Phys. Rev. Letters, 27, 1105 (1971).

[22] J. Gerrat, Mol. Phys., 33, 1199 (1977).

[23] J. Karwowski, Theoret, Chim. Acta, 29, 151 (1973).

[24] J. Karwowski, Chem. Phys. Lett., 19, 279 (1973).

[25] W. Duch, J. Karwowski, Theoret. Chim. Acta, 71, 187 (1987). 
[26] J. Paldus, J. Chem. Phys., 61, 5321 (1974).

[27] J. K. L. McDonald, Phys. Rev., 43, 830 (1933)

[28] W. Duch, GRMS or Graphical Representation ofModel Spaces, Lecture Notes in Chemistry, vol. 42, Springer, Berlin 1986.

[29] C. f! Bender, E. R. Davidson, Phys. Rev., 138, 23 (1969).

[30] E. R. Davidson, Reduced Density Matrices in Quantum Chemistry, Academic Press, New York 1975.

[31] W. Meyer, in Methods ofElectronic Structure Theory, ed. H. F. Schaefer III (Plenum, New York, 1977), p. 413.

[32] R. McWeeny, B. Sutcliffe, Comp. Phys. Reports, 5, 217 (1985).

[33] A. C. Hurley, Electron Correlation in Small Molecules, Academic Press, New York 1976,

[34] P. G. Burton, P. D. Gray, Chem. Phys. Lett., 96, 453 (1983).

[35] B. O. Roos, Intern. J. Quantum Chem., 14, 175 (1980).

[36] P. E. M. Siegbahn et al., Physica Scripta, 21, 323 (1980).

[37] P. E. M. Siegbahn, in The unitary Groupfor the Evaluation of Electronic Energy Matrix Elements, Lecture Notes in Chemistry, vol. 22, ed. J. Hinze (Springer, Berlin 1981) p. 119.

[38] T. Kato, Commun. Pure Appl. Math., 10, 151 (1957).

[39] W. Kutzelnigg, Theoret. Chim. Acta (Berlin), 68, 445 (1985).

[40] W. Klopper, R. Röhse, W. Kutzelnigg, Chem. Phys. Lett., 178, 455 (1991).

[41] J. S. Sims, S. A. Hagstrom, Phys. Rev. A, 4, 908 (1971).

[42] W. Cencek, J. Komasa, J. Rychlewski, J. Chem. Phys., 95, 2572 (1991).

[43] W. Cencek, J. Rychlewski, J. Chem. Phys., 98, 1252 (1993).

[44] N. Flocke, J. Karwowski, Phys. Rev. B, 55, 8287 (1997).

[45] N. Flocke et al., Int. J. Quantum Chem., 61, 1, 11, 21 (1997).

[46] B. O. Roos, Chem. Phys. Lett., 15, 153 (1972).

[47] J. Olsen et al., J. Chem. Phys., 89, 2185 (1988).

[48] M. D. Gould, G. S. Chandler, Intern. J. Quantum Chem., 25, 553; 25, 603 (1984).

[49] G. H. F. Diercksen, G. G. Hall, Comput. Phys., 8, 215 (1994).

[50] S. Wilson, Methods in Computational Chemistry, (Plenum, New York 1987).

[51] S. Wilson, G. H. F. Diercksen, Methods in Computational Molecular Physics, NATO ASI Series, Series B: Physics, vol. 293 (Plenum, New York 1992). 